\title{
Caspases shutdown nonsense-mediated mRNA decay during apoptosis
}

\author{
$\mathrm{J} \mathrm{Jia}^{1,2,3}$, A Furlan ${ }^{1,2,3}$, S Gonzalez-Hilarion ${ }^{4}$, C Leroy ${ }^{1,2,3}$, DC Gruenert ${ }^{5,6}$, D Tulasne ${ }^{1,2,3}$ and F Lejeune ${ }^{\star, 1,2,3}$
}

\begin{abstract}
Nonsense-mediated mRNA decay (NMD) is an mRNA surveillance mechanism that plays integral roles in eliminating mRNAs with premature termination codons to prevent the synthesis of truncated proteins that could be pathogenic. One response to the accumulation of detrimental proteins is apoptosis, which involves the activation of enzymatic pathways leading to protein and nucleic acid cleavage and culminating in cell death. It is not clear whether NMD is required to ensure the accurate expression of apoptosis genes or is no longer necessary since cytotoxic proteins are not an issue during cell death. The present study shows that caspases cleave the two NMD factors UPF1 and UPF2 during apoptosis impairing NMD. Our results demonstrate a new regulatory pathway for NMD that occurs during apoptosis and provide evidence for role of the UPF cleaved fragments in apoptosis and NMD inhibition.
\end{abstract}

Cell Death and Differentiation (2015) 22, 1754-1763; doi:10.1038/cdd.2015.18; published online 6 March 2015

Cell death is a natural process that occurs throughout development and the life of a multicellular organism removing cells that are no longer needed or have become pathogenic, thereby organizing tissues and participating in their homeostasis. There are many pathways leading to cell death that are activated by numerous external and/or internal stimuli. ${ }^{1}$ Cell death can occur via programed processes such as apoptosis, autophagy, necrosis (a more passive process) or necroptosis (a type of programed necrosis). ${ }^{2}$

During apoptosis, specific gene networks and proteincleavage programs are activated sending the cells on a death spiral $^{3-5}$ through a family of cysteine-aspartate proteases (caspases). ${ }^{6}$ Caspases are classified by their role in the apoptotic pathway, into (i) initiator caspases (such as caspases2, 8, 9 and 10) or (ii) effector caspases (such as caspases3, 6 or 7 ). ${ }^{7}$ Initiator caspases cleave the inactive precursor of the effector caspases (pro-caspases) into their active forms. Effector caspases are then responsible for cleaving protein targets to interfere with cellular processes, and in particular, with the activation of some endonucleases that degrade genomic DNA.

Throughout the process of mRNA maturation, numerous quality control mechanisms verify the integrity of the information carried by mRNAs. One of these, nonsense-mediated mRNA decay (NMD), leads to the rapid decay of mRNAs harboring a premature termination codon (PTC) to prevent the synthesis of non-functional and/or potentially detrimental truncated proteins. ${ }^{8-10}$ In addition to its role in quality control, NMD also regulates gene expression of so-called natural substrates of NMD. ${ }^{11-15}$ Proteins that have a central role in
NMD, such as UPF1, UPF2, UPF3/UPF3a and UPF3X/UPF3b are highly conserved from yeast to human. The requirement for these UPF proteins in NMD is illustrated by the fact that the downregulation of any one of them results in an inhibition of NMD. ${ }^{16,17}$ NMD is a necessary component in the development and maintenance of healthy cells and organisms. For example, UPF1 is an essential gene since the inactivation of UPF1 protein leads to an early embryonic death in mouse, ${ }^{18}$ thus implicating NMD as a critical proofreading and/or regulatory component in early organismal development. However, the question of whether NMD is required after cells have committed to proceed along a pathway that culminates in cell death has not been investigated. To address this question, NMD efficiency was studied during apoptosis. The studies presented here show that NMD factors UPF1 and UPF2 are cleaved by caspases 3 and 7 during apoptosis. The functional consequences of these cleavages are a general shutdown of NMD activity leading to stabilization of both PTC-containing mRNAs and natural substrates of NMD, and also the production of caspase-cleaved UPF fragments that induce apoptosis and inhibit NMD.

\section{Results}

UPF proteins are cleaved during apoptosis. Since apoptosis effectors such as caspases target various proteins, ${ }^{19}$ studies were carried out to investigate the possibility that components of the NMD system could themselves be targets. Putative caspase-cleavage sites in UPF proteins were identified using the Support Vector Machine technology for

\footnotetext{
${ }^{1}$ Université de Lille, FRE 3642, Lille, France; ${ }^{2}$ CNRS UMR 8161, Institut de Biologie de Lille, Lille, France; ${ }^{3}$ Institut Pasteur de Lille, Lille, France; ${ }^{4}$ Unité Biologie et Pathogénicité Fongiques, Institut Pasteur, 25 rue du Dr Roux, Paris, France; ${ }^{5}$ Department of Otolaryngology-Head and Neck Surgery, Eli and Edythe Broad Center for Regenerative Medicine and Stem Cell Research, Helen Diller Family Comprehensive Cancer Center, Institute for Human Genetics, Cardiovascular Research Institute, University of California, San Francisco, CA, USA and ${ }^{6}$ Department of Pediatrics, University of Vermont College of Medicine, Burlington, VT, USA

*Corresponding author: F Lejeune, IBL, CNRS, 1 rue du Professeur Calmette, Lille 59019, France. Tel: +333 2087 1059; Fax: +333 2087 1111; E-mail: fabrice.lejeune @ inserm.fr Abbreviations: AA2, apoptosis activator 2; CFP, cyan fluorescent protein; CFTR, cystic fibrosis transmembrane conductance regulator; DMSO, dimethyl sulfoxide; Dpc, day post coitum; EJC, exon junction complex; NMD, nonsense-mediated mRNA decay; mRNA, messenger RNA; mRNP, messenger ribonucleoprotein; PTC, premature termination codon; STS, staurosporine; zVAD-fmk, benzyloxycarbonyl-Val-Ala-DL-Asp-fluoromethylketone

Received 31.7.14; revised 30.1.15; accepted 02.2.15; Edited by B Dynlacht; published online 06.3.15
} 
predicting caspase substrate cleavage sites. $^{20}$ Several putative caspase-cleavage sites are present in human UPF proteins (Supplementary Figure S1). This suggests that these proteins could be targeted by caspases during apoptosis.

To determine if UPF proteins are targeted for degradation during apoptosis, the detection of the full-length proteins and putative UPF cleavage fragments was assessed in apoptotic cells. HeLa cells, incubated with increasing amounts of staurosporine, ${ }^{21,22}$ were assayed for the rate of apoptosis after $24 \mathrm{~h}$ by quantifying the incorporation of annexin $\mathrm{V}$ and propidium iodide into the cells (Figure 1a). This shows that treatment with 2 or $4 \mu \mathrm{M}$ staurosporine results in $\sim 50 \%$ apoptotic cells. At higher staurosporine concentrations, killing was much quicker and more extensive and therefore unsuitable for further NMD efficiency analysis (data not shown). Subsequent studies used $2 \mu \mathrm{M}$ staurosporine as a working concentration combined with a $24 \mathrm{~h}$ exposure.

Next, caspase activation was evaluated by using caspase cleavage of PARP or caspase3 (Figure 1b) and the level of UPF proteins was assessed (Figure 1c). UPF1 and UPF2 protein levels were lower in cells treated with staurosporine compared with those exposed to DMSO. An about $110 \mathrm{kDa}$ UPF1 cleavage fragment was detected in staurosporine-treated extracts using an anti-UPF1 antibody, raised against the C-terminus of UPF1, that could correspond to a C-terminal fragment generated upon cleavage at the putative caspase-cleavage sites $E_{F T D_{37}}$ or $\mathrm{DEED}_{111}$ (Supplementary Figure S1).

Western-blot analysis of UPF2 protein with an antibody recognizing the amino terminal residues identified a new UPF2 isoform of about $75 \mathrm{kDa}$ in staurosporine-treated cells (Figure 1c). This UPF2 isoform could correspond to a $\mathrm{N}$-terminal fragment generated by a cleavage either after DLID $_{585}$ or MHLD M41 (Supplementary Figure S1).

UPF3X level was not affected and additional fragments were not detected by staurosporine treatment even though there are at least two putative canonical caspase-cleavage sites present in this protein (Figure 1c and Supplementary Figure S1). Overall, the results show that staurosporineinduced apoptosis reduced the level of UPF1 and UPF2 proteins suggesting a possible inhibition of NMD during apoptosis. Specifically, the putative UPF1 caspase-cleavage site would separate the $\mathrm{N}$-terminal conserved region of UPF1, shown to be essential for the function of UPF1 in NMD, ${ }^{23}$ from the rest of the protein (Figure 2). Little is known about the role of UPF2 in NMD; however, a recent study has shown that the first and/or the second MIF4G domains of UPF2 are essential for NMD. ${ }^{24}$ Given that the UPF2 caspase-cleavage site is predicted to be located in the second MIF4G making UPF2 nonfunctional during apoptosis (Figure 2).

To identify of the UPF protein species detected by westernblot in the presence of staurosporine, UPF1 or UPF2 were targeted with short interfering RNA (siRNAs) and almost abolished UPF1 or UPF2 related bands, respectively, but not the nonspecific bands (Supplementary Figure S2A).

To evaluate if UPF1 and UPF2 protein cleavage was restricted to staurosporine, other apoptosis activators were assessed (Supplementary Figure S2B). These studies showed that anisomycin, etoposid, AA2 and cisplatin also a

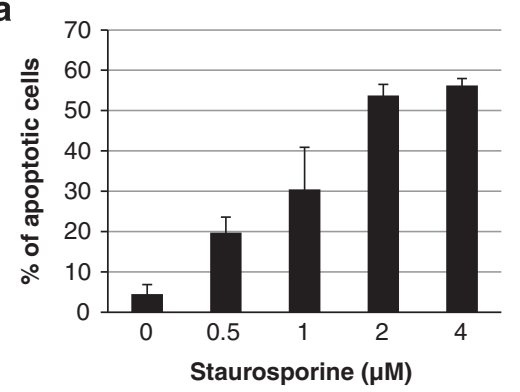

b

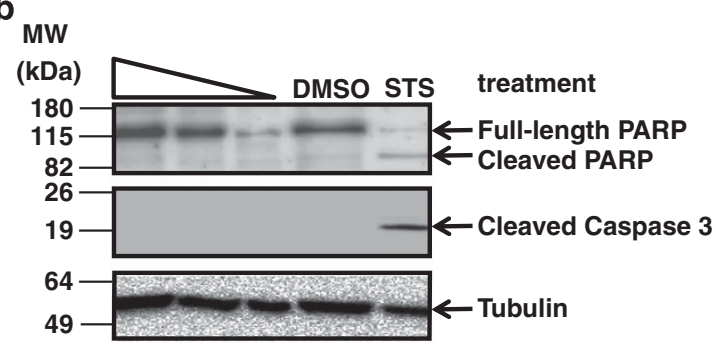

C

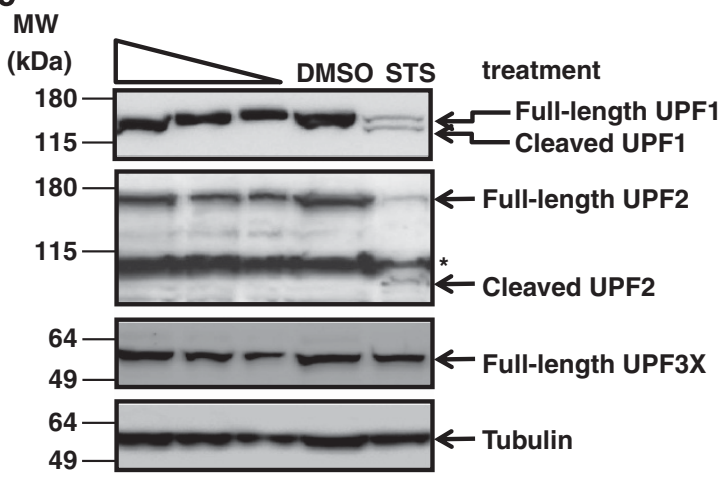

Figure 1 NMD factors UPF1 and UPF2 are cleaved during apoptosis. (a) Determination of the working concentration of staurosporine (STS) to induce apoptosis in HeLa cells. Cells were incubated for $24 \mathrm{~h}$ with increasing amounts of STS before measuring the apoptosis. (b) STS treatment of HeLa cells induces the activation of caspases. The western-blot analysis of PARP or caspase 3 shows the presence of the cleavage product of both proteins demonstrating the activation of caspases, but not of tubulin. (c) Western-blot analysis shows that the level of UPF1 and UPF2, but not UPF3X proteins decreases in cells treated by STS. Cleavage products of UPF1 and UPF2 are indicated on the right side of the figure. UPF1 and UPF2 are degraded during apoptosis; however,UPF3X was not. The three left-most lanes correspond to a two-fold dilution of a wild-type protein extract symbolized by a triangle. The molecular weight scale is presented on the left of all western-blot. The results presented here are representative of three independent experiments. ${ }^{*}$ indicates non-specific protein species

promote cleavage of UPF1 and UPF2, indicating that the effect of staurosporine is a generalized feature of apoptosis inducers (Supplementary Figures S2B and S5).

A caspase inhibitor prevents UPF factors cleavage during apoptosis. To investigate the possible role of caspases in the cleavage of NMD factors UPF1 and UPF2 during apoptosis, HeLa cells were incubated with staurosporine in the presence or in the absence of zVAD-fmk, a pan-caspase inhibitor. As expected, staurosporine-induced 

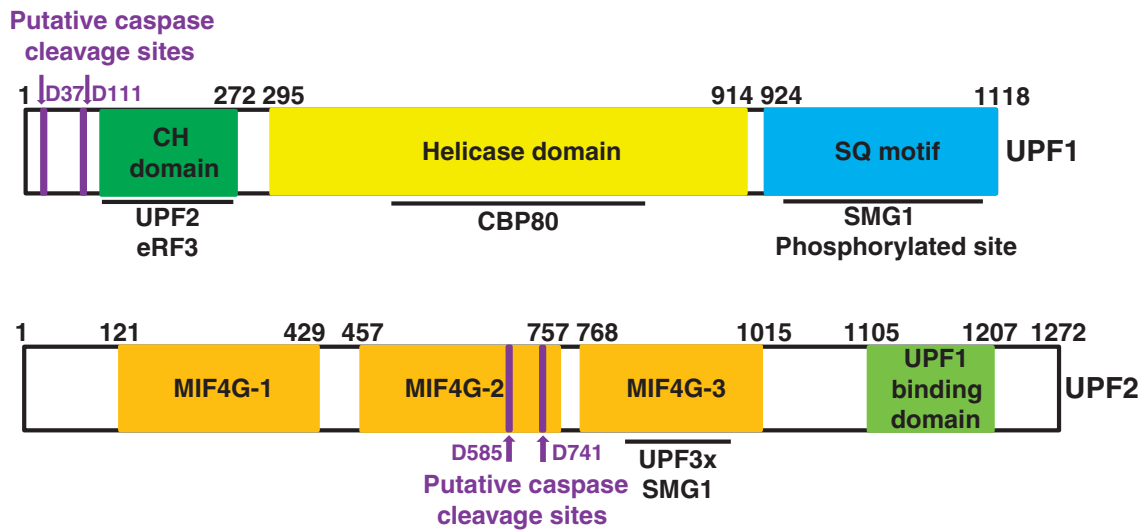

Figure 2 Schematic representation of UPF1 and UPF2 domains. Functional and interacting domains of UPF1 (upper) and UPF2 (lower) are as indicated. The positions of putative caspase-cleavage sites are shown by purple arrows. The amino-acid positions are indicated by a number at the top of each structure

apoptosis and caspase activation were reversed in the presence of the zVAD-fmk (Figure 3b).

To demonstrate that caspases are responsible for the cleavage of NMD factors UPF1 and UPF2 after staurosporine treatment, the levels of UPF1 and UPF2 proteins in cells incubated with or without zVAD-fmk were analyzed by western-blotting (Figure 3c). As expected (Figure 1), staurosporine treatment decreased the levels of UPF1 and UPF2 and showed the appearance of UPF1 and UPF2 cleavage products (Figure 3c). In contrast, in the presence of staurosporine and zVAD-fmk, the levels of UPF1 and UPF2 were almost identical to those in the presence of the DMSO, and no cleavage products were detected (Figure 3c). Overall, these results indicate that caspases are responsible for the cleavage of UPF1 and UPF2 during apoptosis.

Caspase 3 and caspase 7 can cleave UPF1 and UPF2. To identify the caspases responsible for UPF1 and UPF2 cleavage, HeLa-cell extracts were incubated with purified caspases 3, 6, 7, 8 or 9. The results indicate that UPF1 and UPF2 levels were reduced in the presence of caspase3 (Figure 4). Consistent with fragments generated during apoptosis (Figures 1 and 3), a shorter isoform of UPF1 of about $110 \mathrm{kDa}$, and the cleavage product of UPF2 of about $75 \mathrm{kDa}$, were also detected after treatment of the HeLa-cell extract with caspase3.

Although the amount of full-length UPF1 and UPF2 proteins decreased in the presence of caspase7, no cleavage fragments were detected. This suggests that UPF cleavage fragments generated by caspase 7 are unstable. Nevertheless, the results presented in Figure 4 indicate that UPF1 and UPF2 are targeted by caspase 3 and/or caspase 7 suggesting that NMD can be inhibited during apoptosis.

NMD is inhibited during staurosporine- or AA2-induced apoptosis. To characterize the functional consequences of UPF1 and UPF2 cleavage during apoptosis, the efficiency of NMD was first measured in HeLa cells. Cells were transfected with $\beta$-globin gene expression vectors encoding mRNA that was either wild-type (Norm) or harbored a nonsense mutation at codon 39 (Ter) which is an NMD a

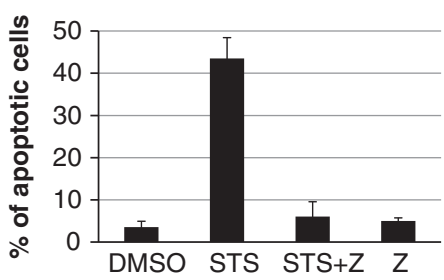

b

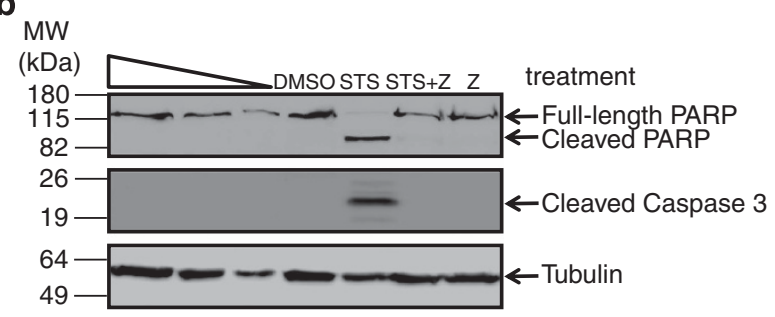

C

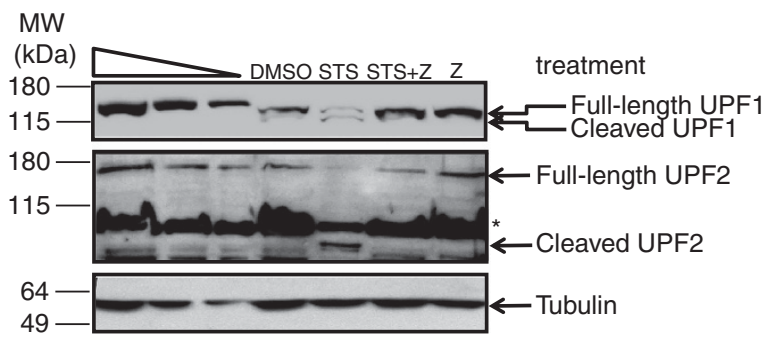

Figure 3 Caspases are responsible for the cleavage of NMD factors UPF1 and UPF2 during apoptosis. (a) Measure of the apoptosis level in cells treated with DMSO, staurosporine (STS), STS and the caspase inhibitor ZVAD-fmk (Z) or Z alone. (b) Western-blot analysis of PARP and caspase 3 shows the presence of cleavage product in the presence of STS, but not in the presence of STS with Z or Z alone. (c) Western-blot analysis of UPF1 or UPF2. Tubulin protein is used as control to ensure that the analysis of each lane can be comparable. * indicates nonspecific protein species. The three most-left lanes of each analysis represent a serial dilution from an untreated cell extract symbolized by a triangle. The protein molecular weight is indicated on the left side of each western-blot analysis. The protein cleavage products are indicated on the right side of the western-blot analysis. Error bar $=$ S.D. Results showed in figure 3 are representative of at least three independent experiments 

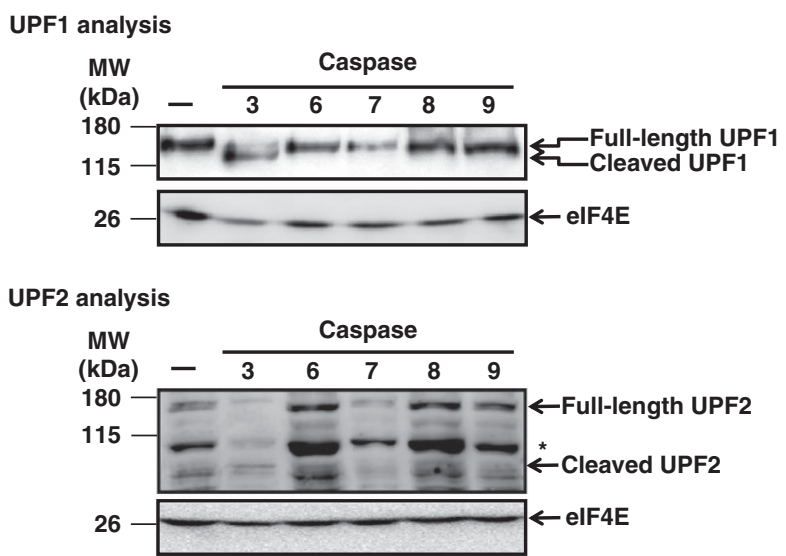

Figure 4 Identification of caspases responsible for the UPF1 or UPF2 cleavage during apoptosis. HeLa cell extract was incubated with purified caspases $3,6,7,8$ or 9 before western-blot analysis for the presence of UPF1 (upper panel) or UPF2 (lower panel). The molecular weight scale is indicated on the left and UPF1 or UPF2 species are shown on the right side of gels. The three left-most lanes correspond to a serial dilution of untreated extract. * indicates nonspecific protein species. The results shown in figure 4 are representative of three independent experiments

target. $^{25,26}$ After a $24 \mathrm{~h}$ exposure to $2 \mu \mathrm{M}$ staurosporine or DMSO, the level of globin Ter mRNA in the cells was about three times higher in the presence of staurosporine than in the presence of DMSO (Figure 5a). These results indicate that NMD is inhibited during apoptosis induced by staurosporine.

The efficiency of NMD during apoptosis was also evaluated in cell lines that produce an endogenous mutated mRNA targeted for NMD. Immortalized cystic fibrosis airway epithelial cells, IB3 harboring the W1282X nonsense mutation in CFTR gene or, alternatively, 6CFSMEo- harboring the Q2X nonsense mutation in CFTR gene (Supplementary Figure S3) 27,28 were also assayed. We first determined that $2 \mu \mathrm{M}$ staurosporine was the lowest concentration that provided efficient apoptosis in each cell line (Supplementary Figure S3A) even though this level of apoptosis is about two-fold less than that obtained in HeLa cells. Under these conditions, CFTR mRNA levels were about 2.5 to 4 fold higher in the presence of staurosporine than in the presence of DMSO (Supplementary Figure S3B). Inhibition of NMD in the presence of staurosporine was even greater than that observed in the presence of amlexanox, a recently identified NMD inhibitor. ${ }^{29}$ To evaluate whether the staurosporine effect observed on PTC-containing mRNAs was not the result of a general increase in transcription, the level of CFTR pre-mRNA was assayed and shown not to significantly change in the presence of staurosporine (Supplementary Figure S4). Overall, these results demonstrate that NMD can be inhibited in several cell-types during staurosporine-induced apoptosis. Due to the higher level of apoptosis measured in HeLa cells when compared with that in IB3 and 6CFSMEo- cells, further experiments were performed in HeLa cells.

Since NMD is inhibited during apoptosis, studies were undertaken to determine whether natural substrates of NMD were also stabilized during apoptosis. The mRNA levels of three different natural NMD substrates (SC35, NAT9 and $\mathrm{TBL} 2)^{11-13}$ were measured in cells incubated with staurosporine or DMSO. The mRNA levels of these substrates were compared to the level of three reference mRNAs that are not subject to NMD (GAPDH, Actin or RPL32). The level of the three natural substrates of NMD showed a two to four-fold of increase compared to the level of the three reference mRNAs (Figure 5b). This result shows that the NMD regulation occurring on natural substrates of NMD is also inhibited during apoptosis.

To demonstrate the involvement of caspases in the inhibition of NMD during apoptosis, NMD efficiency was assessed in the presence of zVAD-fmk. The Figure 6 shows that NMD inhibition under staurosporine treatment was also reversed in the presence of $z V A D$-fmk. It is noteworthy that the presence of the zVAD-fmk alone had no effect on NMD efficiency when apoptosis is not activated, likely because caspases are not in play (Figure 6).

To exclude the possibility that UPF1 and UPF2 cleavage, and NMD inhibition observed during apoptosis is an indirect effect related to staurosporine exposure, NMD was evaluated after exposure to the AA2 apoptosis inducer. ${ }^{30} \mathrm{AA} 2$ activates caspases in a cytochrome c-dependent manner to potentiate apoptosis. After a $16 \mathrm{~h}$ exposure to $50 \mu \mathrm{M}$ AA2 more than $20 \%$ of the HeLa cells went through apoptotic cell death (Supplementary Figure S5A). Under these conditions, the level of exogenous Globin Ter mRNA was about four-fold higher than in vehicle-treated cells (Supplementary Figure S5B). AA2 exposure also caused a decrease of the full-length UPF1 and UPF2 proteins, and the appearance of caspasecleavage products, thereby demonstrating that different inducers of apoptosis can also inhibit NMD (Supplementary Figure S5C).

Caspase cleavage fragments from UPF1 or UPF2 are inducers of apoptosis and/or NMD inhibitors. The presence of cleaved UPF1 or UPF2 fragments during apoptosis in cells treated with staurosporine or AA2 raises the question of their role in NMD inhibition and/or in apoptosis. The specific identity of the caspase-cleavage sites in the UPF1 and UPF2 proteins was determined by mutating aspartic acid to asparagine at positions 37 or 111 in UPF1 and 585 or 741 in UPF2 to block caspase-cleavage. CFP-tagged constructs were used in order to discriminate full-length proteins and their cleavage products from their endogenous counterparts. Overexpression of the wild-type or D111N UPF1 transgene, showed a baseline presence of the UPF1 cleavage fragment that increased with staurosporine. No cleavage fragment was detected with the D37N UPF1 construct (Figure 7a). With regard to UPF2, the caspase-cleavage product was not detected under any conditions by using the CFP-UPF2 constructs for reasons that remain unclear. A decrease in CFP-UPF2 protein was observed with the wild-type or the D585N UPF2 construct, while no decrease was observed with the D741N UPF2 construct (Figure 7b). These results indicate that the caspase-cleavage site is located at position 37 in UPF1 and at position 741 in UPF2 (Figure 7).

To evaluate the impact of $\mathrm{N}$ - or C-terminal UPF1 or UPF2 fragments generated by caspase-cleavage on apoptosis, HeLa cells were transfected with an expression vector encoding a CFP-fusion peptide with the N-terminal part of UPF1 (CFP-UPF1-Nter), C-terminal part of UPF1 
a

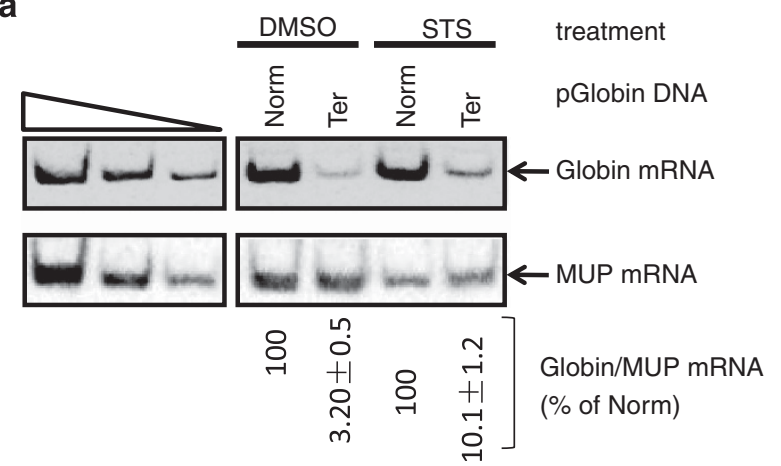

b

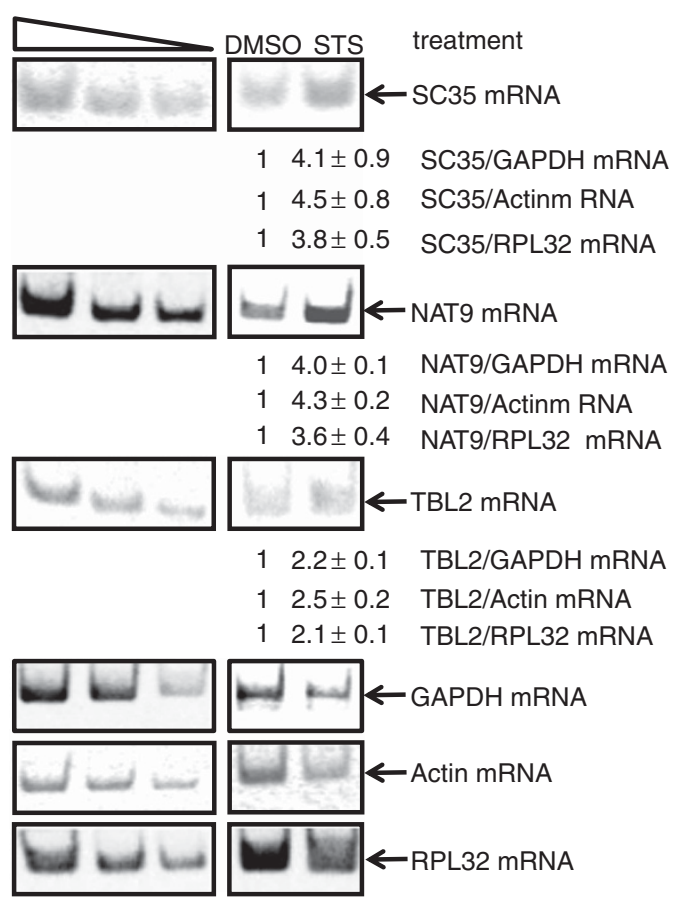

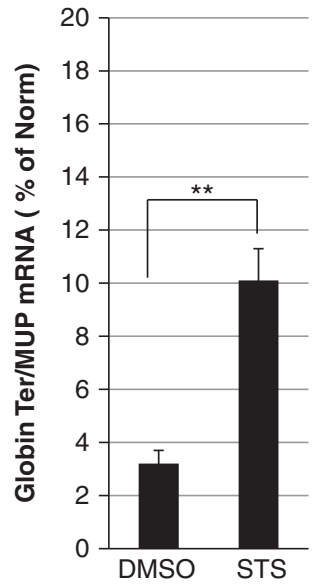

Figure 5 NMD is inhibited during apoptosis. (a) Measure of NMD efficiency as determined by quantitative RT-PCR in HeLa cells transfected with expression vectors for globin (Norm or Ter) and an expression vector for MUP in the presence of DMSO (as a negative control) or staurosporine (STS). The three left-most lanes represent a serial dilution of Norm RT in the PCR. A histogram representation of the results is presented at the right side of the gel. (b) Natural substrates of NMD are upregulated during STS-induced apoptosis. HeLa cells were incubated either with DMSO or with STS for $24 \mathrm{~h}$ before purifying RNAs and measured by quantitative RT-PCR the level of natural NMD substrates SC35, Nat9 or Tbl2 mRNAs. GAPDH, Actin or RPL32 mRNA were used to normalize the level of SC35, Nat9 or Tbl2 mRNA level at each condition as indicated. The three left-most lanes represent a serial dilution of Norm RT in the PCR symbolized by a triangle. Error bar $=$ S.D., student $t$-test: ${ }^{\star \star} P<0.05$. Results are representative of three independent experiments

(CFP-UPF1-Cter), N-terminal part of UPF2 (CFP UPF2-Nter) or the C-terminal part of UPF2 (CFP UPF2-Cter) corresponding to each UPF1 or UPF2 caspase-cleaved fragments. The expression level of each exogenous caspase-cleaved UPF protein fragments was assessed by western-blotting analysis $48 \mathrm{~h}$ after transfection (Supplementary Figure S6). At the expression levels of exogenous UPF1 or UPF2 fragments indicated there was about 10-fold increase in apoptosis in cells expressing the $\mathrm{N}$-terminal caspase-cleavage fragment from UPF1 or UPF2, while cells expressing the C-terminal fragments or the full-length UPF1 or UPF2 proteins showed no such increase (Figure 8a). These results suggest that the $\mathrm{N}$-terminal caspase-cleaved fragment from both UPF proteins promote apoptosis.
To determine the contribution of UPF caspase-cleavage fragments to the rate of staurosporine-induced apoptosis, UPF1 or UPF2 were downregulated with siRNAs (Supplementary Figure S7). Although, UPF1 or UPF2 caspase-cleavage fragments were not detectable in the presence of UPF1 or UPF2 siRNAs, respectively, the rate of staurosporine-induced apoptosis remains unchanged even though the downregulation by itself of UPF1 or UPF2 by siRNAs induces apoptosis (Supplementary Figure S7B), suggesting that the production of UPF1 and UPF2 apoptotic cleavage fragments have only a modest role in cell death.

To assess the effect of the caspase UPF1 and UPF2 cleaved fragments on NMD, HeLa cells were transfected with vectors encoding Globin Norm or Ter, MUP and one of the 

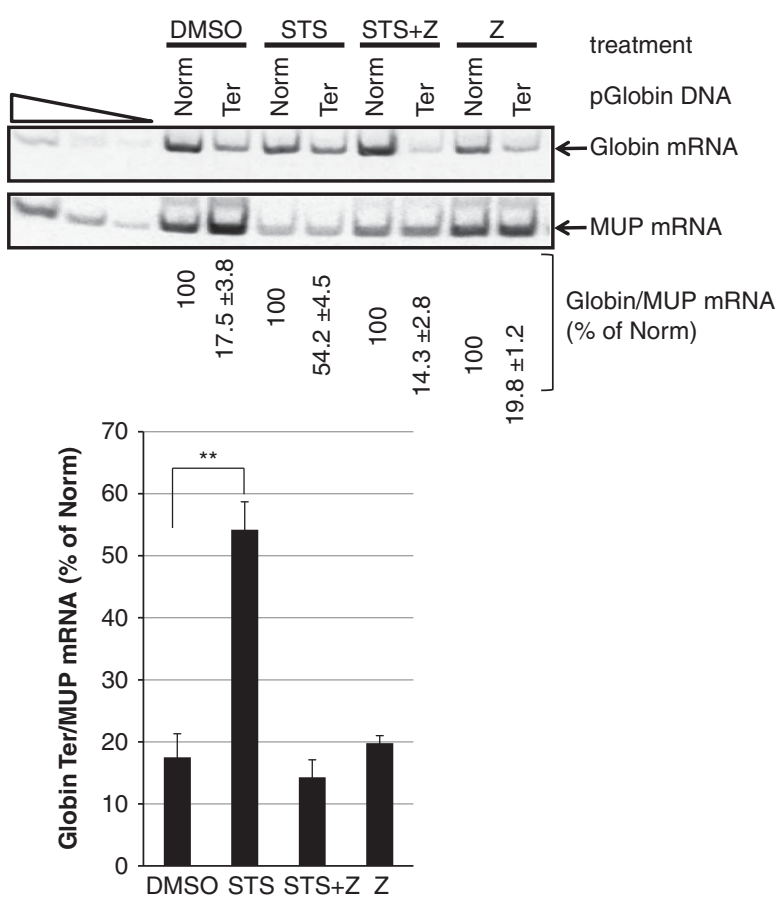

Figure 6 Caspases are responsible for the NMD inhibition during apoptosis. HeLa cells were transfected with expression vectors encoding globin mRNA either WT (Norm) or harboring the 39-PTC (Ter) or MUP mRNA and then incubated with DMSO, staurosporine (STS), STS with zVAD-fmk (Z) or Z alone. The level of globin and MUP mRNA was quantified by RT-PCR.The three left-most lanes represent a serial dilution of Norm RT in the PCR symbolized by a triangle. A histogram representation of the mRNA measures is presented under the gel. Results are representative of three independent experiments. Error bar $=$ S.D., student $t$-test: ${ }^{* *} P<0.05$

caspase-generated $\mathrm{N}$ - or C-terminal fragments, or the fulllength UPF1 or UPF2 (Figure 8b). Assessment of NMD showed about 2-3-folds NMD inhibition in cells expressing either the C-terminal fragment of UPF1 or the $\mathrm{N}$-terminal fragment of UPF2. The dominant negative interference of the C-terminal fragment of UPF1 on NMD is consistent with a previous report. ${ }^{23}$ Interestingly, the N-terminal UPF2 fragment shows the capacity of both inducing apoptosis and inhibiting NMD.

Assessment of NMD inhibition during apoptosis in vivo. Our previous results indicate that NMD is inhibited as a consequence of activating the cell-intrinsic apoptotic pathway. To generalize these results we interrogated whether the activation of the cell-extrinsic (death receptor) apoptotic pathway is also able to inhibit NMD and whether the shutdown of NMD can occur in vivo. Mice were injected with an agonistic anti-Fas antibody to ligate the Fas death receptor and initiate cell-extrinsic apoptosis in hepatocytes as previously described. ${ }^{31}$ The impact of hepatic apoptosis on NMD was assessed through the analysis of liver mRNAs and proteins after anti-Fas antibody exposure. The apoptotic status of hepatocytes was monitored by the presence of cleaved caspase3 (Figure 9a). The in silico analysis of mouse UPF sequences shows that the caspase-cleavage sites identified for UPF1 and UPF2 are conserved between mice

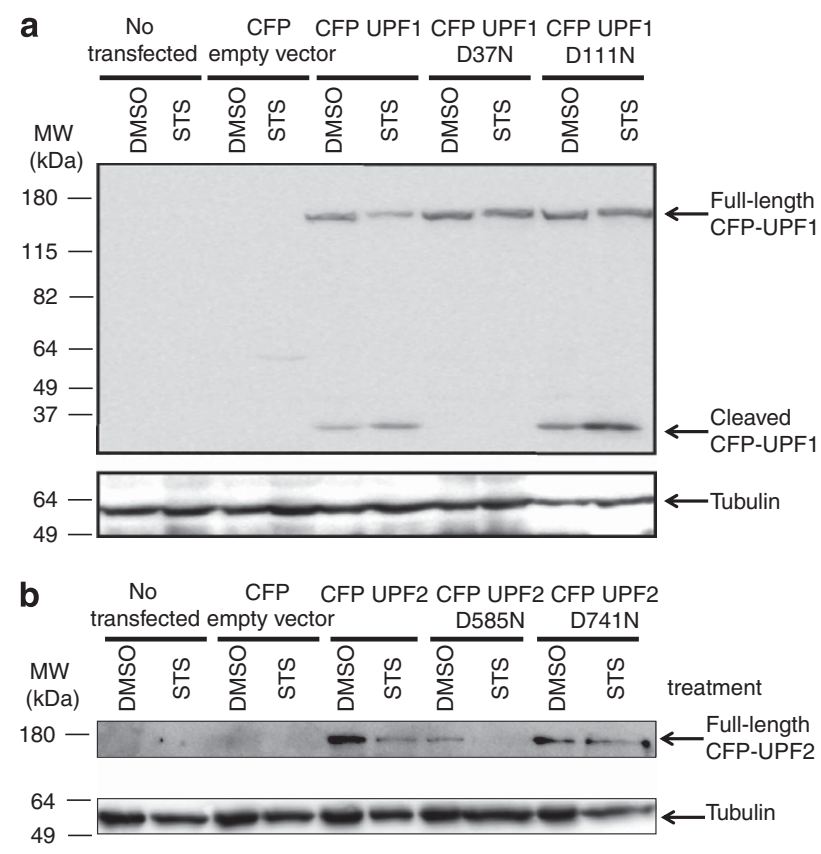

Figure 7 Identification of the caspase-cleavage sites in UPF1 and UPF2. (a) Identification of the caspase-cleavage site in UPF1. Western-blot analysis using an anti-GFP antibody from protein purified from HeLa cells that were either not transfected or transfected with an expression vector encoding the CFP tag, a fusion protein CFP-UPF1 or a fusion protein CFP-UPF1 with a D37N or D111N missense mutation, with or without staurosporine (STS) treatment. (b) Identification of the caspase-cleavage site in UPF2. Western-blot analysis using an anti-GFP antibody from protein purified from HeLa cells that were either not transfected or transfected with an expression vector encoding the CFP tag, a fusion protein CFP-UPF2 or a fusion protein CFP-UPF2 with a D585N or D741N missense mutation, with or without staurosporine (STS) treatment. The molecular weight scale is shown on the left side of each gel and the identity of each species is shown on the right side of each gel. The results presented Figure 7 are representative of 3 independent experiments

and human (Supplementary Figure S1). In apoptotic livers, a decrease was observed in the level of UPF1 protein, without a corresponding decrease in UPF3X (Figure 9a). UPF1 cleavage products were not detected, suggesting that they may be more unstable in vivo than in vitro or that the apoptosis was too massive and the UPF cleavage fragments are already degraded at the time of the cell harvesting. Analysis of UPF2 levels was not possible, since none of the UPF2 antibodies used could detect mouse UPF2 protein (data not shown).

NMD efficiency in mice was measured by exploiting the conservation of the alternative splicing between human and mouse at intron 3 of the mouse Srsf7 gene. ${ }^{15,32}$ The retention of intron 3 in Srsf7 mRNA introduces PTCs recognized by NMD. ${ }^{15,32}$ Quantification of the alternative Srsf7 transcripts in mice exposed to anti-Fas antibody showed a five-fold increase in intron 3-containing mRNA transcript compared with mice injected with saline (Figure 9b). General inhibition of splicing during apoptosis can be excluded since retention of intron 4 in Srsf7 mRNA was not detected in the PCR amplification product encompassing intron 3 to exon 5 used in this analysis (Figure 9b). Overall, the results in Figure 9 indicate that NMD is inhibited during apoptosis both in vitro and in vivo. 

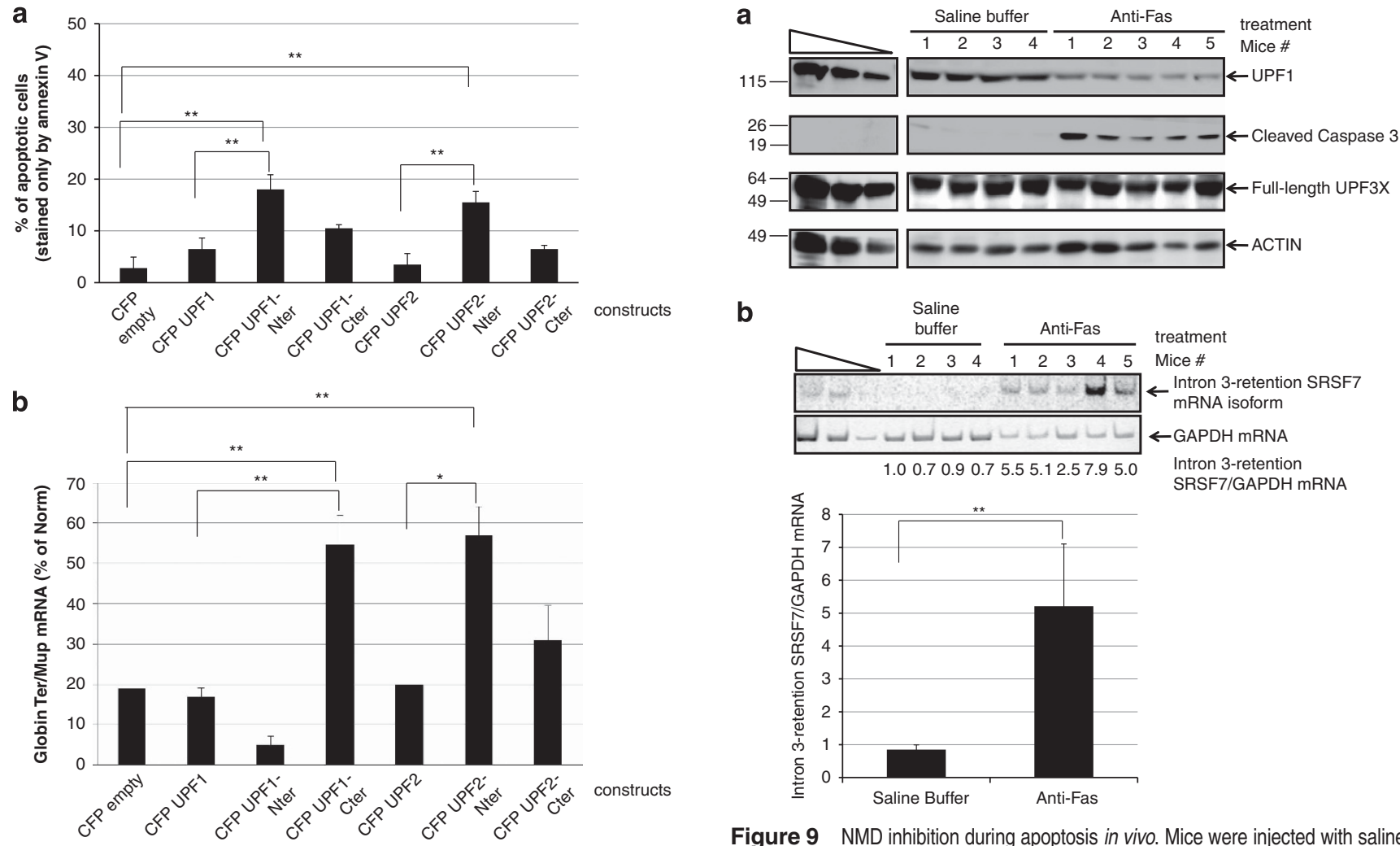

Figure 8 UPF caspase-cleavage fragments induce apoptosis and/or NMD inhibition. (a) Measure of apoptosis as described before in HeLa cells expressing one of the UPF caspase-cleavage fragments or as a control the empty expression vector (CFP) or the full-length UPF protein UPF1 (CFP-UPF1) or UPF2 (CFP UPF2). The $\mathrm{N}$-terminal caspase-cleavage fragment of UPF1, UPF2 or the C-terminal caspasecleavage fragment of UPF1 or UPF2 are noted CFP-UPF1-Nter, CFP UPF2-Nter, CFP-UPF1-Cter or CFP UPF2-Cter, respectively. (b) Effect of the UPF caspasecleavage fragments on NMD efficiency. HeLa cells were transfected with expression vectors encoding globin mRNA Norm or Ter, MUP mRNA as a loading control, one of the UPF caspase-cleavage fragments or as a control the empty expression vector or the expression vector encoding the full-length UPF1 or UPF2. The level of NMD efficiency was measured by RT-PCR after quantifying the level of Globin and MUP mRNA. A histogram representation of the mRNA measures is presented. The results of figure 8 are representative of two independent experiments. Error bar $=S$.D., student $t$-test: ${ }^{*} P<0.1 ;{ }^{*} P<0.05$

\section{Discussion}

During apoptosis, caspases target a wide panel of proteins to promote cell death. In silico prediction programs reveal putative caspase-cleavage sites in UPF proteins encoding key NMD factors (Supplementary Figure S1), and we experimentally demonstrate that UPF1 and UPF2 but not UPF3X are cleaved during staurosporine-induced apoptosis (Figure 1). These cleavages are prevented when the pancaspase inhibitor zVAD-fmk is added in the presence of staurosporine, indicating that caspases are responsible for the cleavage of UPF1 and UPF2 during apoptosis (Figure 3). In vitro results implicate caspase 3 at least in mediating UPF1 and UPF2 cleavage (Figure 4). Due to the central role of UPF proteins in NMD, the functional consequence of the cleavage of NMD factors UPF1 and UPF2 is the inhibition of NMD, as demonstrated for transfected as well as for endogenous

Figure 9 NMD inhibition during apoptosis in vivo. Mice were injected with saline buffer or with anti-Fas antibody $4 \mathrm{~h}$ before harvesting the liver. Proteins were analyzed by western-blot (a) and mRNAs by quantitative RT-PCR (b). The three most-left lanes correspond to a serial dilution of untreated extract symbolized by a triangle. A histogram representation of the mRNA measures of panel $(\mathbf{b})$ is presented under the gel. Error bar $=$ S.D., student $t$-test: ${ }^{* \star} P<0.05$

nonsense mutation-containing genes and natural substrates of NMD in different cell lines (Figure 5 and Supplementary Figure S3). Moreover, staurosporine-induced NMD inhibition was reversed with the pan-caspase inhibitor, zVAD-fmk (Figure 6). These findings are further reinforced by the use of AA2, which confirmed the cleavage of UPF1 and UPF2 (Supplementary Figure S5). Together, these results indicate that both staurosporine and AA2 represent two new nonspecific NMD inhibitors. More importantly, this study demonstrates that the inhibition of NMD observed during apoptosis in cultured cells also extends to tissues undergoing apoptosis in vivo (Figure 9).

It was particularly noteworthy that UPF1 and UPF2 were targeted by caspases, while UPF3X was not, despite the presence of putative caspase-cleavage sites in UPF3X (Supplementary Figure S1). Targeting UPF1 to efficiently inactivate NMD is reasonable since all NMD pathways are unified by their requirement for UPF1, unlike UPF2. ${ }^{33}$ The targeting of UPF2 by caspases suggests that either UPF2 has a more central role in NMD than previously thought or other functions of UPF2 unrelated to its role in NMD must be inhibited for apoptotic cell death to progress. It is possible that there are other NMD-related proteins, such as SMG proteins or EJC components targeted by caspases to ensure the complete arrest of NMD. For example, proteins from the translation initiation complex have been shown to be targeted by caspases during apoptosis to block more than $60 \%$ of the 
protein synthesis. ${ }^{34}$ Among these proteins, elF4G which has a central role in the pioneer round of translation through its interaction with PABPC1 and CBP80, is targeted and inactivated by caspase $3 .^{35}$

The work presented here shows that UPF1 and UPF2 cleavage occurs at positions D37 and D741, respectively (Figure 7) not only to inactivate both NMD factors per se but also to generate fragments that induce apoptosis (Figure 8a). However, this contribution to apoptosis appears to be modest and is likely due to the wide spectrum of caspase targets that influence apoptosis. ${ }^{3-5}$ Further investigations will be necessary to elucidate the mechanism underlying the induction of apoptosis by these UPF cleavage fragments, to identify the proteins that bind these fragments and in particular the highly conserved N-terminal UPF1 fragment for which there is, as yet, no clear function. With respect to UPF2, specific functions have not been correlated with the different regions of UPF2 other than the interaction domain in the $\mathrm{C}$-terminal region with UPF1 and SMG1. ${ }^{24}$ This C-terminal region partially retains the UPF2 function in NMD and is consistent with findings showing a dominant negative effect of the $\mathrm{N}$-terminal caspasecleavage UPF2 fragment on NMD, which is not observed with the C-terminal caspase-cleavage fragment (Figure 8). Our study shows that the $\mathrm{N}$-terminal part of UPF2 has the dual property to induce apoptosis and to inhibit NMD making this region of interest to understand the function of the fulllength UPF2.

During apoptosis, various pathways that ultimately lead to cell death are either inhibited or activated. NMD no longer appears to be required during apoptosis, given that the level of PTC-containing mRNAs increase in the presence of apoptosis activators (Figures 5,6 and 9 and Supplementary Figures S3 and S5). The levels of natural substrates of NMD are also upregulated during apoptosis (Figure 5b and Figure 9b) which could promote apoptosis by allowing the expression of toxic proteins.

In this study, the results show a bidirectional link between NMD and apoptosis in the sense that apoptosis induces NMD inhibition and caspase-cleaved UPF fragments promote apoptosis. The induction of apoptosis by NMD inhibition has previously been demonstrated during embryogenesis in particular. Apoptosis, as well as NMD, is essential during embryogenesis, and is involved in removing unnecessary cells to help shape and define tissues ${ }^{36}$ and organs. NMD is likely involved in degrading inaccurate mRNAs that could interfere with the normal developmental program. In the event of a molecular dysfunction, such as NMD arrest during embryogenesis, apoptosis and eventually embryonic lethality can occur. For instance, the absence of UPF1 in 3.5 days post coitum (dpc) results in blastocysts death by apoptosis, ${ }^{18}$ while the lack of SMG1 leads to embryonic death at day $8.5 \mathrm{dpc}$ by apoptosis. ${ }^{37}$ However, NMD inhibition in vivo does not always lead to activation of apoptosis. This is illustrated by the UPF2 knock-out mice that do not undergo apoptotic embryonic death. ${ }^{38}$ Other examples include NMD inhibition by amlexanox where there is no effect on cell viability, ${ }^{29}$ or more recently, by a new set of NMD inhibitors targeting the interaction between UPF1 and SMG7 proteins with a low cellular toxicity. ${ }^{39}$ Whether NMD inhibition can activate apoptosis might be dependent on the degree of NMD inhibition.
The data presented here demonstrate that apoptosis regulates NMD in addition to other already identified molecular pathways such as miRNAs, Staufen-mediated mRNA decay or the response to stress (for review see Karam et al. ${ }^{10}$ ). The data also raise the question of whether there are other cell death pathways that promote NMD inhibition. For example, apoptotic-like programed cell death does not involve caspases activation, but rather an apoptosis-inducing factor. ${ }^{40}$ Recent studies have also suggested that inhibition of NMD can lead to autophagy; ${ }^{41}$ however, whether NMD is inhibited during distinct autophagic inductions has yet to be determined. Cell death by necrosis is yet another mechanism that involves a family of proteases, the calpains rather than caspases. It would therefore be of interest to assess whether NMD is also active during these other cell death pathways.

\section{Materials and Methods}

Cell culture. HeLa cells were incubated in DMEM supplemented with $10 \%$ fetal bovine serum (FBS) and ZellShield (Minerva Biolabs, Berlin, Germany). IB3 cells were grown in LHC-8 supplemented with 10\% FBS and ZellShield, and 6CFSMEo-27,28,42 and $16 \mathrm{HBE} 140-42,43$ were grown in $\alpha$-MEM supplemented with $10 \% \mathrm{FBS}, 1 \mathrm{mM}$ L-glutamine and ZellShield. Chemical treatment of the cells involved: Staurosporine (Sigma-Aldrich, Lyon, France)-at $2 \mu \mathrm{M}$ for $24 \mathrm{~h}$; Z-VAD-FMK (Santa Cruz Biotechnology, Dallas, TX, USA) $-20 \mu \mathrm{M}$ added 30 min before incubating the cells with staurosporine; amlexanox (Sequoia Research Products Limited, Pangbourne, UK) $-25 \mu \mathrm{M}$ for $24 \mathrm{~h}$; apoptosis activator 2 (Santa Cruz Biotechnology) $-50 \mu \mathrm{M}$ for $16 \mathrm{~h}$; Anisomycin (Santa Cruz Biotechnology) at $50 \mu \mathrm{M}$, Etoposide (Sigma-Aldrich) at $50 \mu \mathrm{M}$, or Cisplatin (Mylan, Saint Priest, France) at $50 \mu \mathrm{g} / \mathrm{ml}$ for $24 \mathrm{~h}$.

qRT-PCR analysis. qRT-PCR analysis was performed as previously described. ${ }^{29,44}$ The list of primer sequences used in this study is: Globin (sense: 5'GGACGAGCTGTACAAGTATC3', antisense:5'GGGTTAGTGGTACTTGTGAGC3'), MUP (sense: 5'CTGATGGGGCTCTATG3', antisense: 5'TCCTGGTGAGAAGTC TCC3'), CFTR mRNA (sense: 5'GGCCAGAGGGTGGGCCTCTT3', antisense: 5'AGGAAACTGTTCTATCACAG3'), CFTR pre-mRNA (sense: 5'TTGATGCC TAGAGGGCAGAT3', antisense 5'TGTCAAAGGGATTGGGAGGG3'), SC35 (sense: 5'CCTCTTAAGAAAATGCTGCGGTCTC3', antisense: 5'ATCAGCCAAATCAGTT AAAATCTGC3'), NAT9 (sense: 5'ATTGTGCTGGATGCCGAGA3', antisense: 5' ACCTAGCGTGGTCACTCCGTA3'), TBL2 (sense: 5'GCAGTCATTTACCACATGC3', antisense: 5'TATTGTTTCTGCTTCTTGGAT3'), GAPDH (sense: 5'CATTGACCT CAACTACATGG3', antisense: 5'GCCATGCCAGTGAGCTTCC3'), Actin (sense: 5'ATGAGGTAGTCAGTCAGGTCCC3', antisense: 5'CAGAAGGATTCCTATGTG GGCG3'), RPL32(sense:5'TTGACAACAGGGTTCGTAG3', antisense: 5'TTCTTGGAG GAAACATTGTG3'), mouse SRSF7 (sense (intron 3): 5'CTTCGTTTGAGTCAGT CGCC3', antisense (exon 5): 5'AAGCTGATCTTGATCTACG3').

Western-blot analysis. Proteins were extracted in lysis buffer $(50 \mathrm{mM}$ Tris pH7, $20 \mathrm{mM}$ EDTA and 5\% SDS) and then loaded on a 10\% SDS-PAGE (except UPF1 which was loaded on 6\% SDS-PAGE). After migration, proteins were transferred to a nitrocellulose membrane. The membrane was incubated with a primary antibody overnight at $4^{\circ} \mathrm{C}$ and then with a secondary antibody coupled to HRP activity (Jackson Immuno Research, West Grove, PA, USA) at $4^{\circ} \mathrm{C}$ for $2 \mathrm{~h}$. Finally, proteins were detected using SuperSignal West Femto Maximum Sensitivity Substrate (Pierce-Biotechnology, Rockford, IL, USA). Primary antibodies used were: rabbit anti-hUPF1 at 1/5000 (Abcam, Paris, France), rabbit anti-hUPF2 at 1/5000 (antibody raised against the following synthetic peptide: H2N-MPA ERK KPA SME EKD C-CONH2 (Eurogentec, Angers, France)), rabbit anti-UPF3X at 1/5000 (Aviva Systems Biology, San Diego, CA, USA), rabbit anti-PARP at 1/1000 (Santa Cruz Biotechnology), rabbit cleaved Caspase-3 (Asp175) at 1/1000 (Cell Signaling Technology, Leiden, The Netherlands), rabbit anti-tubulin at 1/1000 (Epitomics, Burlingame, CA, USA), mouse anti-elF4E at 1/200 (Santa Cruz Biotechnology) and rabbit anti-GFP at 1/5000 (Sigma-Aldrich).

Apoptosis assays. Apoptosis was measured according to the manufacturer's protocol (Life Technologies, Saint Aubin, France) using the Tali Apoptosis Kitcontaining annexin V Alexa Fluor 488 and propridium iodide. Measurements were 
performed on a Tali cytometer (Life Technologies). Apoptotic cells were identified as the cells incorporating only annexin V.

In vivo apoptosis assay. Mouse experiments were performed according to institutional guidelines. Briefly, 6-week old male C57BL/6 mice, fasted overnight and were then intraperitoneally injected with the anti-Fas J02 antibody (BD Pharmingen, San Diego, CA, USA) at $0.2 \mu \mathrm{g} / \mathrm{g}$ body weight or saline buffer vehicle as a control. After $4 \mathrm{~h}$, mice were euthanized and livers were harvested.

Caspase cleavage reaction. Assessment of caspase-cleavage was as previously described. ${ }^{45}$ Briefly, $3 \times 10^{5} \mathrm{HeLa}$ cells/well were distributed in 6 wellplates. After $24 \mathrm{~h}$, cells were lysed in $80 \mu \mathrm{l}$ of the lysis buffer ( $20 \mathrm{mM}$ Pipes pH7.2, $100 \mathrm{mM} \mathrm{NaCl}, 1 \%$ Chaps, $10 \%$ Sucrose $+5 \mathrm{mMDTT}+50 \mu \mathrm{M}$ EDTA). Cell extracts were then incubated with $1 \mu \mathrm{l}$ of purified caspase for $4 \mathrm{~h}$ at $37^{\circ} \mathrm{C}$ before loading on a $10 \%$ SDS-PAGE for western-blot analysis. Purified, active caspases were generously provided by $\mathrm{Dr}$ GS Salvesen (The Burnham institute, La Jolla, CA, USA). The concentration of purified caspase 3 is $13.9 \mu \mathrm{M}, 7.5 \mu \mathrm{M}$ for caspase 6 , $19.7 \mu \mathrm{M}$ for caspase $7,15 \mu \mathrm{M}$ for caspase 8 and $41.5 \mu \mathrm{M}$ for caspase 9 .

Construction of the plasmids expressing UPF fragments. UPF1 derived plasmids: CFP-UPF1 CDNA was introduced into pUNO vector (Cayla, Toulouse, France) linearized by BamHI using the Infusion cloning kit (Clontech, Mountain View, CA, USA). CFP-UPF1 was amplified by PCR with primer for CFP: sense: 5'AGATCACCGGCGTGTCGACGATGGTGAGCAAGGGCGAGGA3'; antisense: 5'CGTACGCCTCCACGCTCATCTTGTACAGCTCGTCCATGCC3'; primers for UPF1: sense: 5'ATGAGCGTGGAGGCGTACG3'; antisense: 5'TGGCTGCAGA GCGCTGGATCTTAATACTGGGACAGCC3'). The same method was used to amplify CFP-UPF1Nter (amino-acid 1-37) using the same primer sense as for CFP-UPF1 and the antisense primer: 5'TGGCTGCAGAGCGCTGGATCTTAG TCGGTGAACTCGAACTCGG3'), and CFP-UPF1Cter (amino-acid 38-1118) was amplified using the sense primer: 5'TTTACTCTTCCTAGCCAGACG3' and the same antisense primer as for CFP-UPF1.

UPF2 derived plasmids were constructed according to Gateway cloning technology (Life Technologies) using empty vector pSPO-CFP-rfc. The list of primers used to amplify UPF2 full length or fragments is: for the full length UPF2 (called as CFP UPF2 expressing amino acid from 1-1272) (sense: 5'GGGGACAAGTTTGTACAAAAAAGCAGGCTCCATGCCAGCTGAGCGTAAAAAG CCAG3', antisense: 5'GGGGACCACTTTGTACAAGAAAGCTGGGTTCAACGTC TCCTCCCACCAGTCTTA3'), the N-terminal fragment of UPF2 (called as CFP UPF2-Nter expressing amino acids from 1-741) (sense: $5^{\prime}$ GGGGACA AGTTTGTACAAAAAAGCAGGCTCCATGC CAGCTGAGCGTAAAAAGCCAG', antisense: 5 ' GGGG ACCACTTTGTACAAGAA AGCTGG GT ATCAAGATGC ATTGCTTGCTTCTTT $3^{\prime}$ ), the C-terminal fragment of UPF2 (called as CFP UPF2Cter expressing amino acids from 742-1272) (sense: $5^{\prime}$ GGGGACAAGTTTGTACAA AAAAGCAGGCTCCGCGAGATACGTCACAATGGTAGAGA 3', antisense: 5' GGGG ACCACTTTGTACAAGAAAGCTGGGTTCAACGTCTCCTCCCACCAGTCTTA $3^{\prime}$ ).

Construction of mutant UPF plasmids. Aspartic acid was changed to asparagine using QuikChange Site-Directed Mutagenesis Kit (Stratagene, La Jolla, CA, USA) and the following primers: UPF1 D37N (sense: $5^{\prime}$ CCGAGTTC GAGTTCACCAACTTTACTCTTCCTAGC3'; antisense: 5' GCTAGGAAGAGTAAA GTTGGTGAACTCGAACTCGG3'); UPF1 D111N (sense: 5'CTTCGAGGAAGATG AAGAAAACACCTATTACACGAAGG3'; antisense: 5'CCTTCGTGTAATAGGTGTTTT CTTCATCTTCCTCGAAG3') UPF2 D585N (sense: 5'CAACCGAGATCTGATA AACAAGGCAGCAATGGATTTTTGC3'; antisense 5'GCAAAAATCCATTGCTGCC TTGTTTATCAGATCTCGGTTG3'), UPF2 D741N (sense: 5' GCAAGCAATGCATC TTAATGCGAGATACGTCACAATGG3'; antisense: 5' CCATTGTGACGTATCTCGCA TTAAGATGCATTGCTTGC3').

UPF knockdown by siRNA. HeLa cells were transfected with the ICAFectin 442 reagent (In Cell Art, Nantes, France) complexed with either $100 \mathrm{nM}$ of siControl (Eurogentec), or with siUPF1 (5'-AAGATGCAGTTCCGCTCCATTTT-3') or siUPF2 (5'-GAAGTTGGTACGGGCACTC-3'). At $24 \mathrm{~h}$ post-transfection, cells were incubated with DMSO or $2 \mu \mathrm{M}$ of staurosporine for an additional $24 \mathrm{~h}$. The cells were then collected to measure apoptosis by AnnexinV or were extracted in lysis buffer for western-blot analysis.

\section{Conflict of Interest}

The authors declare no conflict of interest.
Acknowledgements. We thank Dr. Jérome Vicogne for helpful discussions and Pr. Lynne Maquat, Dr Edouard Bertrand and Dr. GS Salvesen for reagents and comments on the paper. We also thank the mouse facility of Institut Pasteur de Lille. $\mathrm{JJ}$ is funded by Vaincre la mucoviscidose. FL received fundings for this project from Vaincre la mucoviscidose, l'Association Française contre les myopathies and la Fondation ARC. DCG was supported by funds from Pennsylvania Cystic Fibrosis, Inc. and Cystic Fibrosis Research, Inc.

\section{Author contributions}

JJ, AF, SGH, CL and FL performed experiments. JJ, AF, CL, DT, DCG and FL wrote the manuscript. JJ, AF, SGH, CL, DCG, DT ad FL designed the experiments and analyzed data.

1. Elmore S. Apoptosis: a review of programmed cell death. Toxicol Pathol 2007; 35: 495-516.

2. Galluzzi L, Vitale I, Abrams JM, Alnemri ES, Baehrecke EH, Blagosklonny MV et al. Molecular definitions of cell death subroutines: recommendations of the Nomenclature Committee on Cell Death 2012. Cell Death Differ 2012; 19: 107-120.

3. Reimertz C, Kogel D, Rami A, Chittenden T, Prehn JH. Gene expression during ER stressinduced apoptosis in neurons: induction of the $\mathrm{BH} 3$-only protein Bbc3/PUMA and activation of the mitochondrial apoptosis pathway. J Cell Biol 2003; 162: 587-597.

4. Zhang XJ, Yang L, Zhao Q, Caen JP, He HY, Jin QH et al. Induction of acetylcholinesterase expression during apoptosis in various cell types. Cell Death Differ 2002; 9: 790-800.

5. Johnson NA, Sengupta S, Saidi SA, Lessan K, Charnock-Jones SD, Scott L et al. Endothelial cells preparing to die by apoptosis initiate a program of transcriptome and glycome regulation. FASEB J 2004; 18: 188-190.

6. Alnemri ES, Livingston DJ, Nicholson DW, Salvesen G, Thornberry NA, Wong WW et al. Human ICE/CED-3 protease nomenclature. Cell 1996; 87: 171.

7. Frejlich E, Rudno-Rudzinska J, Janiszewski K, Salomon L, Kotulski K, Pelzer $\mathrm{O}$ et al. Caspases and their role in gastric cancer. Adv Clin Exp Med 2013; 22: 593-602.

8. Popp MW, Maquat LE. The dharma of nonsense-mediated mRNA decay in Mammalian cells. Mol Cells 2014; 37: 1-8.

9. Muhlemann $O$, Eberle AB, Stalder L, Zamudio Orozco R. Recognition and elimination of nonsense mRNA. Biochim Biophys Acta 2008; 1779: 538-549.

10. Karam R, Wengrod J, Gardner LB, Wilkinson MF. Regulation of nonsense-mediated mRNA decay: implications for physiology and disease. Biochim Biophys Acta 2013; 1829: 624-633.

11. Mendell JT, Sharifi NA, Meyers JL, Martinez-Murillo F, Dietz HC. Nonsense surveillance regulates expression of diverse classes of mammalian transcripts and mutes genomic noise. Nat Genet 2004; 36: 1073-1078.

12. Viegas MH, Gehring NH, Breit S, Hentze MW, Kulozik AE. The abundance of RNPS1, a protein component of the exon junction complex, can determine the variability in efficiency of the nonsense mediated decay pathway. Nucleic Acids Res 2007; 35: 4542-4551.

13. Sureau A, Gattoni R, Dooghe Y, Stevenin J, Soret J. SC35 autoregulates its expression by promoting splicing events that destabilize its mRNAs. EMBO J 2001; 20: 1785-1796.

14. Wollerton MC, Gooding C, Wagner EJ, Garcia-Blanco MA, Smith CW. Autoregulation of polypyrimidine tract binding protein by alternative splicing leading to nonsensemediated decay. Mol Cell 2004; 13: 91-100.

15. Lareau LF, Inada M, Green RE, Wengrod JC, Brenner SE. Unproductive splicing of SR genes associated with highly conserved and ultraconserved DNA elements. Nature 2007; 446: 926-929.

16. Mendell JT, ap Rhys CM, Dietz HC. Separable roles for rent1/hUpf1 in altered splicing and decay of nonsense transcripts. Science 2002; 298: 419-422

17. Chan WK, Bhalla AD, Le Hir H, Nguyen LS, Huang L, Gecz J et al. A UPF3-mediated regulatory switch that maintains RNA surveillance. Nat Struct Mol Biol 2009; 16: 747-753.

18. Medghalchi SM, Frischmeyer PA, Mendell JT, Kelly AG, Lawler AM, Dietz HC. Rent1, a transeffector of nonsense-mediated mRNA decay, is essential for mammalian embryonic viability. Hum Mol Genet 2001; 10: 99-105.

19. Shi Y. Caspase activation, inhibition, and reactivation: a mechanistic view. Protein Sci 2004; 13: 1979-1987.

20. Wee LJ, Tan TW, Ranganathan S. SVM-based prediction of caspase substrate cleavage sites. BMC Bioinform 2006; 7: S14.

21. Koh JY, Wie MB, Gwag BJ, Sensi SL, Canzoniero LM, Demaro J et al. Staurosporineinduced neuronal apoptosis. Exp Neurol 1995; 135: 153-159.

22. Zwelling LA, Altschuler E, Cherif A, Farquhar D. N-(5,5-diacetoxypentyl)doxorubicin: a novel anthracycline producing DNA interstrand cross-linking and rapid endonucleolytic cleavage in human leukemia cells. Cancer Res 1991; 51: 6704-6707.

23. Kashima I, Yamashita A, Izumi N, Kataoka N, Morishita R, Hoshino S et al. Binding of a novel SMG-1-Upf1-eRF1-eRF3 complex (SURF) to the exon junction complex triggers Upf1 phosphorylation and nonsense-mediated mRNA decay. Genes Dev 2006; 20: 355-367.

24. Clerici M, Deniaud A, Boehm V, Gehring NH, Schaffitzel C, Cusack S. Structural and functional analysis of the three MIF4G domains of nonsense-mediated decay factor UPF2. Nucleic Acids Res 2013; 42: 2673-2686.

25. Zhang J, Sun X, Qian Y, Maquat LE. Intron function in the nonsense-mediated decay of betaglobin mRNA: indications that pre-mRNA splicing in the nucleus can influence mRNA translation in the cytoplasm. RNA 1998; 4: 801-815. 
26. Thermann R, Neu-Yilik G, Deters A, Frede U, Wehr K, Hagemeier C et al. Binary specification of nonsense codons by splicing and cytoplasmic translation. EMBO J 1998; 17: 3484-3494.

27. Cozens AL, Yezzi MJ, Chin L, Simon EM, Finkbeiner WE, Wagner JA et al. Characterization of immortal cystic fibrosis tracheobronchial gland epithelial cells. Proc Natl Acad Sci USA 1992; 89: 5171-5175.

28. da Paula AC, Ramalho AS, Farinha CM, Cheung J, Maurisse R, Gruenert DC et al Characterization of novel airway submucosal gland cell models for cystic fibrosis studies. Cell Physiol Biochem 2005; 15: 251-262.

29. Gonzalez-Hilarion S, Beghyn T, Jia J, Debreuck N, Berte G, Mamchaoui K et al. Rescue of nonsense mutations by amlexanox in human cells. Orphanet J Rare Dis 2012; 7: 58

30. Nguyen JT, Wells JA. Direct activation of the apoptosis machinery as a mechanism to target cancer cells. Proc Natl Acad Sci USA 2003; 100: 7533-7538.

31. Lefebvre J, Muharram G, Leroy C, Kherrouche Z, Montagne R, Ichim G et al. Caspasegenerated fragment of the Met receptor favors apoptosis via the intrinsic pathway independently of its tyrosine kinase activity. Cell Death Dis 2013; 4: e871.

32. Lejeune F, Cavaloc Y, Stevenin J. Alternative splicing of intron 3 of the serine/arginine-rich protein $9 \mathrm{G} 8$ gene. Identification of flanking exonic splicing enhancers and involvement of $9 \mathrm{G} 8$ as a trans-acting factor. J Biol Chem 2001; 276: 7850-7858.

33. Gehring NH, Kunz JB, Neu-Yilik G, Breit S, Viegas MH, Hentze MW et al. Exon-junction complex components specify distinct routes of nonsense-mediated mRNA decay with differential cofactor requirements. Mol Cell 2005; 20: 65-75.

34. Clemens MJ, Bushell M, Jeffrey IW, Pain VM, Morley SJ. Translation initiation factor modifications and the regulation of protein synthesis in apoptotic cells. Cell Death Differ 2000; 7: 603-615.

35. Marissen WE, Lloyd RE. Eukaryotic translation initiation factor $4 G$ is targeted for proteolytic cleavage by caspase 3 during inhibition of translation in apoptotic cells. Mol Cell Biol 1998; 18: $7565-7574$.
36. Suzanne M, Steller H. Shaping organisms with apoptosis. Cell Death Differ 2013; 20: 669-675.

37. Mcllwain DR, Pan Q, Reilly PT, Elia AJ, McCracken S, Wakeham AC et al. Smg1 is required for embryogenesis and regulates diverse genes via alternative splicing coupled to nonsense-mediated mRNA decay. Proc Natl Acad Sci USA 2010; 107: 12186-12191.

38. Thoren LA, Norgaard GA, Weischenfeldt J, Waage J, Jakobsen JS, Damgaard I et al UPF2 is a critical regulator of liver development, function and regeneration. PLOS One 2010; 5: e11650.

39. Martin L, Grigoryan A, Wang D, Wang J, Breda L, Rivella S et al. Identification and characterization of small molecules that inhibit nonsense mediated RNA decay and suppress nonsense p53 mutations. Cancer Res 2014; 74: 3104-3113.

40. Susin SA, Zamzami N, Castedo M, Hirsch T, Marchetti P, Macho A et al. Bcl-2 inhibits the mitochondrial release of an apoptogenic protease. J Exp Med 1996; 184: 1331-1341.

41. Wengrod J, Martin L, Wang D, Frischmeyer-Guerrerio P, Dietz HC, Gardner LB. Inhibition of nonsense-mediated RNA decay activates autophagy. Mol Cell Biol 2013; 33: 2128-2135.

42. Gruenert DC, Willems M, Cassiman JJ, Frizzell RA. Established cell lines used in cystic fibrosis research. J Cyst Fibros 2004; 3: 191-196.

43. Cozens AL, Yezzi MJ, Kunzelmann K, Ohrui T, Chin L, Eng K et al. CFTR expression and chloride secretion in polarized immortal human bronchial epithelial cells. Am J Respir Cell Mol Biol 1994; 10: 38-47.

44. Durand S, Cougot N, Mahuteau-Betzer F, Nguyen CH, Grierson DS, Bertrand E et al. Inhibition of nonsense-mediated mRNA decay (NMD) by a new chemical molecule reveals the dynamic of NMD factors in P-bodies. J Cell Biol 2007; 178: 1145-1160.

45. Foveau B, Leroy C, Ancot F, Deheuninck J, Ji Z, Fafeur V et al. Amplification of apoptosis through sequential caspase cleavage of the MET tyrosine kinase receptor. Cell Death Differ 2007; 14: 752-764

\section{Supplementary Information accompanies this paper on Cell Death and Differentiation website (http://www.nature.com/cdd)}

